\title{
Clinical Utility of Procalcitonin for Differentiating between Cryptogenic Organizing Pneumonia and Community-Acquired Pneumonia
}

\author{
Satoshi Takeda ${ }^{1}$, Nobuhiko Nagata ${ }^{1}{ }^{*}$, Hiroyuki Miyazaki ${ }^{1}$, Takanori Akagi ${ }^{1}$, Taishi Harada $^{1}$, \\ Masaru Kodama ${ }^{1}$, Shinichiro Ushijima ${ }^{1}$, Takashi Aoyama1, Kentaro Wakamatsu' ${ }^{2}$, \\ Masaki Fujita ${ }^{3}$, Kentaro Watanabe ${ }^{3}$ \\ ${ }^{1}$ Department of Respiratory Medicine, Fukuoka University Chikushi Hospital, Chikushino, Japan \\ ${ }^{2}$ Department of Respiratory Medicine, National Hospital Organization Omuta Hospital, Omuta, Japan \\ ${ }^{3}$ Department of Respiratory Medicine, Faculty of Medicine, Fukuoka University, Fukuoka, Japan \\ Email: ${ }^{*}$ bnagata@fukuoka-u.ac.jp
}

Received 13 April 2015; accepted 1 June 2015; published 4 June 2015

Copyright (C) 2015 by authors and Scientific Research Publishing Inc.

This work is licensed under the Creative Commons Attribution International License (CC BY). http://creativecommons.org/licenses/by/4.0/

(c) (i) Open Access

\begin{abstract}
Background: Differentiating cryptogenic organizing pneumonia (COP) from community-acquired pneumonia (CAP) can be difficult in some cases. Objective: To clarify the clinical utility of procalcitonin (PCT) levels for differentiating between COP and CAP. Methods: Blood PCT levels, leukocyte count, C-reactive protein concentration, number of segments involved on computed tomography (CT) images, and pneumonia severity assessment scale were retrospectively investigated from clinical charts and chest CT images of COP and CAP patients who were admitted to our hospital from 2012 to 2014. The clinical characteristics of COP patients were compared to those of CAP patients for whom causative organisms were not detected. Results: There were 16 COP and 94 CAP patients for whom causative organisms were not detected. Blood PCT levels of all COP patients were less than $0.16 \mathrm{ng} / \mathrm{dL}$, and significantly lower than those of CAP patients $(p=0.0004)$, while the number of involved segments was significantly higher than that of CAP patients $(p=$ 0.0001). Blood PCT levels and the number of involved segments remained independently significant for differentiating between COP and CAP by multivariate analysis. Receiver operating characteristics curve analysis revealed that 7 was the best cut-off number for involved segments to differentiate between COP and CAP patients with low PCT levels (sensitivity 85.7\%, specificity 94.7\%). Conclusion: A combination of PCT levels and number of involved segments on CT images is useful for differentiation between COP and CAP.

*Corresponding author.

How to cite this paper: Takeda, S., Nagata, N., Miyazaki, H., Akagi, T., Harada, T., Kodama, M., Ushijima, S., Aoyama, T., Wakamatsu, K., Fujita, M. and Watanabe, K. (2015) Clinical Utility of Procalcitonin for Differentiating between Cryptogenic Organizing Pneumonia and Community-Acquired Pneumonia. International Journal of Clinical Medicine, 6, 372-376.

http://dx.doi.org/10.4236/ijcm.2015.66048
\end{abstract}




\section{Keywords}

\section{Cryptogenic Organizing Pneumonia, Procalcitonin, Community-Acquired Pneumonia, Computed Tomography}

\section{Introduction}

Patients with cryptogenic organizing pneumonia (COP) present with fever, infiltrative shadow on chest roentgenogram, and increased white blood cell count (WBC) or C-reactive protein (CRP) [1] [2]. Therefore, their clinical features resemble those of patients with community-acquired pneumonia (CAP), and many of these patients are, in fact, initially treated with antibiotics. A diagnosis of COP is made based on the presence of airspace filling by plugs of granulation tissue by transbronchial or surgical lung biopsy [1] [2]. However, obtaining biopsies can be difficult in some cases due to various reasons, such as severe hypoxemia, poor general condition, old age, or patient refusal.

Procalcitonin (PCT) is a biomarker specifically elevated in systemic bacterial infections [3]-[7] and has been shown to differentiate bacterial infections from other inflammatory conditions [8]. Therefore, PCT may have clinical utility in the differential diagnosis of COP and CAP. To our knowledge, there was only one report which examined PCT levels of patients with organizing pneumonia (OP). Kolditz et al. reported that PCT levels of OP patients were significantly lower than those of CAP patients, and all of OP patients showed bilateral infiltrates in chest imaging, in contrast to unilateral infiltrate of most CAP patients [9].

This study aimed to clarify the clinical utility of PCT for differentiating between COP and CAP.

\section{Patients and Methods}

Clinical charts and chest computed tomography (CT) images of all COP and CAP patients admitted to our hospital from April 2012 to December 2014 were retrospectively examined. Diagnoses of COP and CAP were reevaluated based on the consensus of two respiratory physicians. Diagnostic criteria of COP included the following: presence of intra-alveolar organization and absence of histologic findings inconsistent with those in COP (such as destruction of alveolar structure, necrosis, granuloma, or aggregates of neutrophils in TBLB); solitary or multifocal infiltrates found predominantly in the peribronchovascular or subpleural areas via CT; no microorganisms detected from respiratory specimens (including sputum, bronchial washing, or bronchoalveolar lavage fluid); and improvement of abnormal shadows with corticosteroid treatment. CAP was defined as an acute illness which occurred outside the hospital setting, and was associated with at least one of the following signs or symptoms: fever, new cough with or without sputum production, pleuritic chest pain, dyspnea, or altered breath sounds on auscultation, in addition to a chest radiograph showing an opacity compatible with the presence of acute pneumonia. Among CAP patients examined, those in whom a causative organism was not detected in sputum or by blood examination (pathogen unknown) were selected for comparison with COP patients. This is the patient population that presents difficulties in differentiating from COP patients. Blood PCT levels, leukocyte count, and CRP concentration examined at admission were investigated from clinical charts, and the number of segments involved on CT images taken within 24 hours of admission was also examined by two of the authors. The severity of pneumonia was assessed by the A-DROP scoring system, which is based on Japanese Respiratory Society guidelines. The ADROP scale is similar to the CURB-65 scale and provides a five-point score based on the following parameters: confusion, blood urea nitrogen $>21 \mathrm{mg} / \mathrm{dL}$ or the presence of dehydration, percutaneous oxygen desaturation $\left(\mathrm{SpO}_{2}<90 \%\right)$, systolic blood pressure $<90 \mathrm{mmHg}$, and age $>70$ years for men or $>75$ years for women. Each criterion receives one point, resulting in a total score ranging from 0 to 5 points [10]. The system was also applied to COP patients. This study was approved by the Institutional Review Board of the Fukuoka University Chikushi Hospital.

\section{Statistical Analysis}

Data are presented as the median (interquartile range). The Mann-Whitney U test was used for comparisons between the two groups. Multivariate analysis was performed with logistic regression analysis. Receiver operating 
characteristic (ROC) curves were generated to determine the optimal cut-off for the number of involved segments in patients whose PCT levels were $<0.25 \mathrm{ng} / \mathrm{mL}$. The optimal cut-off value was obtained from the best sensitivity/specificity ratio. Statistical analyses were performed with Excel Tokei 2010 (Social Survey Research Information, Co., Ltd., Tokyo, Japan). p $<0.05$ was considered statistically significant.

\section{Results}

\subsection{Study Population}

The clinical characteristics of patients are summarized in Table 1 . The study population consisted of 16 (male/ female $=10 / 6)$ COP and 94 (male/female = 62/32) CAP patients. Median (interquartile range) age, PCT, WBC, CRP, number of involved segments, and ADROP score of COP, and CAP patients were 77.0 (69.0 - 78.0) years, and $74.5(69.0$ - 80.3) years; $0.05(0.05-0.07) \mathrm{ng} / \mathrm{mL}$, and $0.27(0.11$ - 1.59) ng/mL; $8000(5700-8200) / \mu \mathrm{L}$, and $9700(7500-12,800) / \mu \mathrm{L} ; 6.28(1.34$ - 8.97) $\mathrm{mg} / \mathrm{dL}$, and 9.83 (5.49 - 18.64) mg/dL; 8.0 (7.0 - 11.0), and 4.0 (2.0 - 5.0); and $1(1-2)$, and $2(1-2)$, respectively. Blood PCT levels of all COP patients were less than 0.16 $\mathrm{ng} / \mathrm{dL}$.

\subsection{Parameters Which Differentiate COP from CAP Patients}

Of the parameters examined, PCT $(\mathrm{p}=0.0004)$, WBC $(\mathrm{p}=0.0093)$, and number of involved segments $(\mathrm{p}=$ 0.0001 ) were significantly different between COP and CAP patients (Table 1). Difference of CRP was marginal $(p=0.0567)$. Multivariate analysis revealed that PCT levels and the number of involved segments were independent factors that differed between COP and CAP patients (OR, 0.0000; 95\% CI, $0.0000-0.1566 ; \mathrm{p}=0.0229$ for PCT levels; OR, 2.1254; 95\% CI, 1.2918 - 3.4969; $\mathrm{p}=0.0030$ for number of involved segments; Table 2). Multivariate analysis including CRP in addition to PCT levels, WBC, and the number of involved segments also showed that PCT levels and the number of involved segments were independent factors (odds ratio (OR), 0.0000; 95\% confidence interval (CI), 0.0000 - 0.1926; $\mathrm{p}$ = 0.0264 for PCT levels; OR, 2.2030; 95\% CI, 1.2699 - 3.8219; $\mathrm{p}=0.0050$ for number of involved segments).

\subsection{Cut-Off for Number of Involved Segments Which Differentiate COP from CAP Patients with Low PCT Levels}

We determined the optimal cut-off for the number of involved segments which differentiates COP from CAP patients whose PCT levels were $<0.25 \mathrm{ng} / \mathrm{mL}$ using ROC curves. Seven was found to be the optimal cut-off (sensitivity, 85.7\%; specificity, 94.7\%; AUC, 0.945; Figure 1).

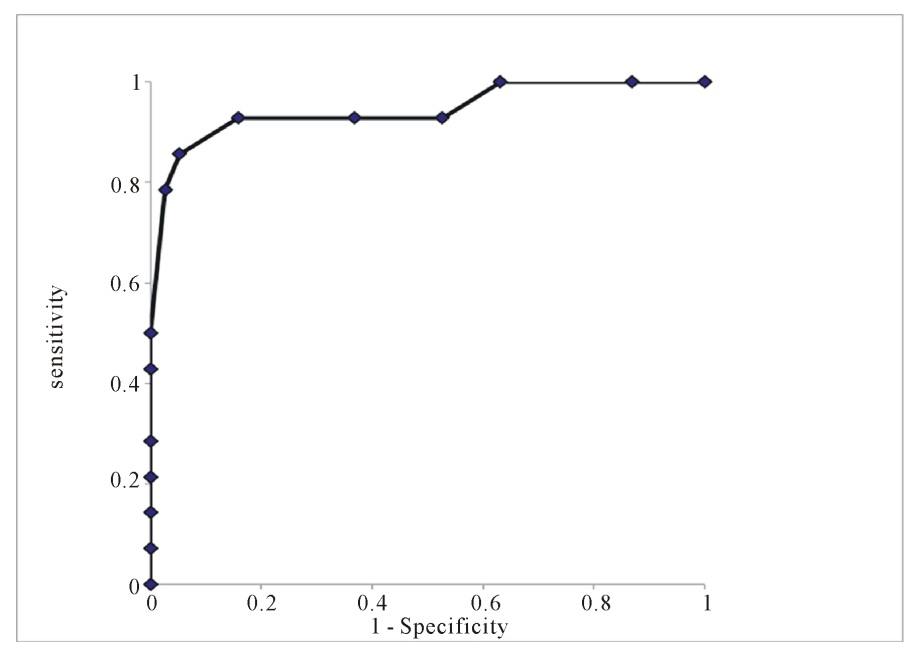

Figure 1. Receiver operating characteristics curve of the number of involved segments for prediction of cryptogenic organizing pneumonia as compared with community-acquired pneumonia with blood procalcitonin levels $<0.25 \mathrm{ng} / \mathrm{mL}$. 
Table 1. Baseline characteristics of patients with COP, and CAP.

\begin{tabular}{cccc}
\hline & COP & CAP & p value COP vs CAP \\
\hline Number of patients & 16 & 94 & \\
Age, years & $77.0(69.0-78.0)$ & $74.5(69.0-80.3)$ & 0.2285 \\
Male/female & $10 / 6$ & $62 / 32$ & 0.5369 \\
PCT, ng/mL & $0.05(0.05-0.07)$ & $0.27(0.11-1.59)$ & 0.0004 \\
WBC, $/ \mu \mathrm{L}$ & $8000(5700-8200)$ & $9700(7500-12800)$ & 0.0093 \\
CRP, mg/dL & $6.28(1.34-8.97)$ & $9.83(5.49-18.64)$ & 0.0567 \\
Number of involved segments & $8.0(7.0-11.0)$ & $4.0(2.0-5.0)$ & 0.0001 \\
ADROP score & $1(1-2)$ & $2(1-2)$ & 0.2856
\end{tabular}

COP: cryptogenic organizing pneumonia; CAP: community-acquired pneumonia; PCT: procalcitonin; WBC: white blood cell; CRP: C-reactive protein; ADROP: age, dehydration, respiration, orientation, and blood pressure. Data are presented as number or median (interquartile range).

Table 2. Multivariate analysis of parameters which differentiate definite COP from CAP.

\begin{tabular}{cccc}
\hline & Odds ratio & 95\% Confidence interval & p value \\
\hline PCT, $\mathrm{ng} / \mathrm{mL}$ & 0.0000 & $0.0000-0.1566$ & 0.0229 \\
WBC, $/ \mu \mathrm{L}$ & 0.9998 & $0.9994-1.0002$ & 0.3591 \\
Number of involved segments & 2.1254 & $1.2918-3.4969$ & 0.0030 \\
\hline
\end{tabular}

COP: cryptogenic organizing pneumonia; CAP: community-acquired pneumonia; PCT: procalcitonin; WBC: white blood cell.

\section{Discussion}

PCT is considered the most useful laboratory marker for differentiating causes of fever, particularly in autoimmune, autoinflammatory, and malignant diseases [8]. Fever is the most prevalent symptoms noted in both CAP and COP patients [1] [2]. In fact, many COP patients are initially considered to have CAP and are treated with antibiotics. COP is a non-infectious, inflammatory condition, and patients with this condition are predicted to have low serum PCT levels. Though blood levels of other inflammatory markers such as WBC and CRP were also different between COP and CAP patients, multivariate analysis revealed that only PCT levels among inflammatory markers examined were independent factors that differed between COP and CAP patients.

Although a diagnosis of COP is confirmed by histological findings of polypoid granulation tissue in the airspace [1] [2], it is sometimes difficult to perform biopsies for various reasons, such as poor general or respiratory status, old age, and patient refusal. In such situations, a decision must be made as to whether to administer corticosteroids. Our present findings apply to such situations, i.e., low serum PCT levels and $>7$ involved segments (multilobar infiltrates) predict a favorable response to corticosteroids. Nonetheless, histological confirmation of COP remains of great importance.

Although PCT is useful for discriminating between infectious and non-infectious fever [8], some CAP patients also exhibit low serum PCT levels. A cut-off of 7 involved segments by CT allowed for the discrimination of COP from CAP patients with low PCT levels and unknown causative pathogen with $85.7 \%$ sensitivity and 94.7\% specificity. This suggests that the combination of low serum PCT levels and $>7$ involved segments favors a diagnosis of COP over CAP. We chose CAP patients for whom the causative pathogen was unknown for comparison with COP patients because these are the patients that present diagnostic difficulties when differentiating CAP from COP.

Kolditz et al. conducted a similar investigation as the present study [9], in which 15 histologically confirmed COP patients were compared with 15 CAP patients matched by age, sex, and disease severity. Their results were similar to ours, although the nature of CAP patients differed from that of the present study. We build on their findings by demonstrating that the number of involved segments on CT images can help discriminate COP patients from CAP patients who do not exhibit elevated serum PCT levels.

There are several limitations to this study. First, the number of COP patients was small. Second, the study was retrospective in nature. Although a prospective study that includes a large number of COP patients will be 
needed to confirm our results, the sporadic occurrence of COP may render a prospective study design difficult.

\section{Conclusion}

COP patients exhibit significantly lower PCT levels and a significantly higher number of involved segments on CT images compared to CAP patients. A combination of PCT levels and number of involved segments on CT images is useful for differentiation between COP and CAP.

\section{Author Contributions}

ST and NN contributed to the planning of the study, data analysis, and preparation, review, and submission of the manuscript. TA, TH, MK, SU, AS, and KW contributed to data collection, data analysis, and review and submission of the manuscript. MF and KW contributed to data analysis and review and submission of the manuscript.

\section{Conflict of Interest}

None to report.

\section{References}

[1] Cordier, J.-F. (2006) Cryptogenic Organizing Pneumonia. European Respiratory Journal, 28, 422-446. http://dx.doi.org/10.1183/09031936.06.00013505

[2] Cottin, V. and Cordier, J.F. (2012) Cryptogenic Organizing Pneumonia. Seminars in Respiratory and Critical Care Medicine, 33, 462-475. http://dx.doi.org/10.1055/s-0032-1325157

[3] Goros, C.A., Drosou, E., Bassaris, H.P. and Skoutelis, A. (2000) Pro- versus Anti-Inflammatory Cytokine Profile in Patients with Severe Sepsis: A Marker for Prognosis and Future Therapeutic Options. Journal of Infectious Diseases, 181, 176-180. http://dx.doi.org/10.1086/315214

[4] Christ-Crain, M. and Mueller, B. (2005) Procalcitonin in Bacterial Infections-Hype, Hope, More or Less? Swiss Medical Weekly, 135, 451-460.

[5] Christ-Crain, M. and Mueller, B. (2007) Biomarkers in Respiratory Tract Infections: Diagnostic Guides to Antibiotic Prescription, Prognostic Markers and Mediators. European Respiratory Journal, 30, 556-573. http://dx.doi.org/10.1183/09031936.00166106

[6] Linscheid, P., Seboek, D., Zulewski, H., Keller, U. and Muller, B. (2005) Autocrine/Paracrine Role of InflammationMediated Calcitonin Gene-Related Peptide and Adrenomedullin Expression in Human Adipose Tissue. Endocrinology, 146, 2699-2708. http://dx.doi.org/10.1210/en.2004-1424

[7] Schuetz, P., Christ-Crain, M. and Mueller, B. (2009) Procalcitonin and Other Biomarkers to Improve Assessment and Antibiotic Stewardship in Infection-Hope for Hype? Swiss Medical Weekly, 139, 318-326.

[8] Limper, M., de Kruif, M.D., Duits, A.J., Brandjes, D.P.M. and van Gorp, E.C.M. (2010) The Diagnostic Role of Procalcitonin and Other Biomarkers in Discriminating Infectious from Non-Infectious Fever. Journal of Infection, 60, 409-416. http://dx.doi.org/10.1016/j.jinf.2010.03.016

[9] Kolditz, M., Halank, M., Schulte-Hubbert, B. and Höffken, G. (2012) Procalcitonin Improves the Differentiation between Infectious and Cryptogenic/Secondary Organizing Pneumonia. Journal of Infection, 64, 122-124. http://dx.doi.org/10.1016/j.jinf.2011.10.012

[10] Yanagihara, K., Kohno, S. and Matsushima, T. (2001) Japanese Guidelines for the Management of Community-Acquired Pneumonia. International Journal of Antimicrobial Agents, 18, S45-S48. http://dx.doi.org/10.1016/S0924-8579(01)00402-2 